\title{
Maternal docosahexaenoic acid supplementation during pregnancy and visual evoked potential development in term infants: a double blind, prospective, randomised trial
}

\author{
C A Malcolm, D L McCulloch, C Montgomery, A Shepherd, L T Weaver
}

Arch Dis Child Fetal Neonatal Ed 2003;88:F383-F390

See end of article for authors' affiliations

Correspondence to: Dr Malcolm, Department of Nursing and Midwifery, University of Stirling, Stirling FK9 4LA, Scotland, UK:

gcl305@clinmed.gla.c.uk

Accepted

30 October 2002

\begin{abstract}
Aim: To test the hypothesis that maternal docosahexaenoic acid (DHA) supplementation during pregnancy enhances maturation of the visual evoked potential (VEP) in healthy term infants.

Methods: One hundred women were supplemented with either fish oil capsules rich in DHA ( $n=50$ ) or placebo capsules ( $n=50$ ) from week 15 of pregnancy until delivery. Total fatty acids in red blood cells and plasma were measured at weeks 15,28, and 40 of pregnancy and at delivery in umbilical cord blood. Infant visual pathway development was assessed using VEPs recorded to flash stimuli shortly after birth and to both flash and pattern-reversal stimuli at 50 and 66 weeks post-conceptional age (PCA).

Results: Maternal supplementation did not significantly elevate the level of DHA in umbilical cord blood. Moreover, there were no significant differences in any of the VEP measures observed between supplementation groups. However, maturity of the pattern-reversal VEP at 50 and 66 weeks PCA was associated with DHA status of the infants at birth. Infants with higher DHA status, both as a concentration and as a percentage of total fatty acids, showed shorter P100 peak latencies of the pattern-reversal VEP than those with lower DHA status.

Conclusions: Maternal DHA supplementation during pregnancy did not enhance VEP maturation in healthy term infants. However, these results show an association between the DHA status of infants at term and early postnatal development of the pattern-reversal VEP, suggesting that DHA status itself may influence maturation of the central visual pathways.
\end{abstract}

$\mathrm{T}$ he long chain polyunsaturated fatty acids (LCPUFAs), in particular docosahexaenoic acid (DHA), are important for optimal development of the visual and nervous systems. ${ }^{1}$ The grey matter of the visual cortex and the photoreceptor outer segment membranes of the retina are highly enriched in DHA. ${ }^{23}$ Considerable quantities of LCPUFAs accumulate in the brain and retina during the final trimester of pregnancy and the initial months of life. ${ }^{45}$ It is essential that the fetus and neonate receive adequate amounts of DHA during these critical developmental periods. The human fetus, however, has a limited ability to synthesise DHA from its precursor, $\alpha$ linolenic acid, and therefore it must be largely supplied from maternal sources. ${ }^{6-8}$ Maternal supplies of DHA are transferred to the fetus through the placenta and to the neonate from breast milk. A progressive decline in maternal LCPUFA status during pregnancy has been observed.' Infants born of multiparous mothers have lower DHA levels than those of primiparous mothers, and maternal DHA stores are not fully replenished in the interval between successive pregnancies. ${ }^{10}$ As the major source of DHA to the developing fetus, maternal LCPUFA status during pregnancy plays a role in determining the essential fatty acid status of the neonate at birth. There is evidence of higher LCPUFA status at birth in infants born to mothers who were supplemented with fish oils during pregnancy. ${ }^{11} 12$

A considerable body of research has been conducted over recent years to evaluate the functional effects of a dietary supply of DHA, in either supplemented formulas or breast milk, on the visual development of preterm and term infants. Electrophysiological tests, such as the visual evoked potential (VEP) and electroretinogram and/or behavioural measures of vision have been used to assess retinal and visual pathway maturation in these studies. Most supplementation studies in preterm infants have suggested improved retinal ${ }^{13}{ }^{14}$ and visual function ${ }^{15-20}$ in those infants fed DHA supplemented formulas or breast milk. Results of dietary supplementation studies in term infants have proved to be less consistent. Some studies have shown improved visual acuity development in term infants fed breast milk or DHA supplemented formulas, ${ }^{21-23}$ whereas others have shown no significant effect of DHA supplementation on visual acuity development. ${ }^{24-27}$

Most previous studies have examined the effects of supplementing infant diets with DHA on various measures of visual function. To our knowledge, this is the first report in which maternal diet during pregnancy was supplemented and DHA levels of mothers and infants were compared with visual pathway maturation.

A previous study of non-pregnant women in the Glasgow area defined the DHA status of non-pregnant women of reproductive age, and revealed that this population consumes low levels of fish, particularly oily fish. ${ }^{28}$ A supplemental maternal supply of DHA during gestation should increase maternal DHA status, and thereby increase the amount of DHA available to the fetus, thus enhancing postnatal DHA status of mother and infant. Moreover, an enhanced supply of DHA during the last trimester of pregnancy, when the visual and nervous systems are in a state of rapid growth and maturation, could enhance visual development. Therefore the aim

Abbreviations: DHA, docosahexaenoic acid; VEP, visual evoked potential; RBC, red blood cell; PCA, post-conceptional age; \% TFA, percentage of total fatty acids; LCPUFA, long chain polyunsaturated fatty acid 
Table 1 Timetable showing times of supplementation, sampling, and vision testing

\begin{tabular}{|c|c|c|c|c|c|}
\hline & 15 weeks & 28 weeks & Birth* & $\begin{array}{l}50 \text { weeks } \\
\text { PCA }\end{array}$ & $\begin{array}{l}66 \text { weeks } \\
\text { PCA }\end{array}$ \\
\hline Fatty acid supplements & \multicolumn{5}{|c|}{$\begin{array}{l}\ldots \text { Fish oil capsules } \\
\ldots \ldots \ldots \text { Placebo capsules . . . }\end{array}$} \\
\hline Venous blood & - & - & - & & \\
\hline Umbilical cord blood & & & $\diamond$ & & \\
\hline Breast milk & & & - & - & - \\
\hline Flash VEP & & & 口 & 口 & ㅁ \\
\hline Pattern-reversal VEP & & & & 口 & $\square$ \\
\hline Anthropometry & - & & ㅁ & $\square$ & 口 \\
\hline Diet assessement (fish intake) & - & - & $-\square$ & $-\square$ & $\square$ \\
\hline
\end{tabular}

of this study was to test the hypothesis that dietary supplementation of pregnant women with the LCPUFA, DHA, affects visual pathway maturation of the term infant as assessed by the flash and pattern-reversal VEP.

\section{METHODS \\ Design}

This study was double blind, prospective, randomised, and controlled in design. Ethical approval was granted from the ethics committee of Yorkhill NHS Trust, and written informed consent was obtained from each study participant at the time of enrollment. One hundred pregnant women were recruited to the study at about week 15 of pregnancy, while attending an antenatal clinic for an elective $\alpha$ fetoprotein test. Inclusion criteria included women who were expected to deliver their infants at term and planned to feed them on breast and/or formula milk. Women with diabetes, twin pregnancies, pre-eclampsic toxaemia, a past history of abruption or postpartum haemorrhage, allergy to fish products, a thrombophilic tendency, or who were receiving drugs that affect thrombocyte function (non-steroidal anti-inflammatories), were excluded.

At study entry, information on age, previous obstetric history, smoking habits and alcohol intake, exercise patterns, and dietary intake of fish was collected from the participants through a questionnaire. Socioeconomic status was measured using the Carstairs score. ${ }^{29}$ The women were then randomised to either the treatment group, where they would receive a DHA rich fish oil supplement, or the control group, where they would receive a high oleic acid sunflower oil placebo for the remainder of their pregnancy. A venous blood sample was obtained at study entry to determine baseline maternal DHA status.

The women were requested to continue taking the dietary supplements daily until delivery. At week 28 of pregnancy and delivery, each participant was interviewed to monitor compliance and assess dietary intake of additional sources of DHA. Compliance in capsule consumption was monitored by asking the women to return their capsule bottles and counting the number of capsules missing and the time period over which they were consumed to determine an approximate ingested dosage. ${ }^{30}$ The women were asked to report the frequency and type of fish products consumed in an average week as well as additional sources of dietary DHA, including vitamin and/or herbal supplements. A maternal venous blood sample was also obtained at these time points. Samples of umbilical cord blood were obtained at delivery. Women were asked to report any fish products consumed within the 24-36 hours before sample collection.

Only those infants born after 36 weeks gestation, with an Apgar score of greater than seven at five minutes and without any detectable visual, medical, or developmental disorders were eligible to continue in the postnatal phase of the study. Visual pathway maturation was assessed using transient flash and pattern-reversal VEPs at three separate time points. The first session took place as soon as possible after birth (one to five days) and before the mothers and infants were discharged from hospital. The second session took place at 50 weeks postconceptional age (PCA), and the third and final session at 66 weeks PCA, approximately equal to 10 weeks and 6 months of age respectively. The visual system matures at a rapid rate during the early months of life. Therefore every effort was made to test the infants as close as possible to the chosen target ages. The VEP tests were performed within five days after delivery, within \pm 1 week of 50 weeks PCA and within \pm 2 weeks of 66 weeks PCA. In addition to the VEP tests, anthropometric assessments of the infants were performed. If the infants were being breast fed at any of these time points, a sample of breast milk was also collected. The mothers were asked to provide information on the infants' diet including method of feeding, age at which breast feeding was discontinued, and type of formula and/or weaning foods. Table 1 gives the timetable for supplementation, sampling, and infant vision testing.

\section{Dietary supplements}

The dose of DHA chosen for supplementation was $200 \mathrm{mg}$ a day, similar to those used in previous supplementation studies of pregnant women. ${ }^{31}$ The study capsules, both fish oil and placebo, were provided by R P Scherer Limited (Swindon, Wiltshire, UK). The fish oil capsule consisted of a blended fish oil, Marinol D40, and contained $100 \mathrm{mg}$ DHA in $323 \mathrm{mg}$ oil per capsule. The placebo capsule contained $323 \mathrm{mg}$ sunflower oil with high levels of oleic acid and was free of any significant amounts of LCPUFAs or their precursors. Table 2 outlines the fatty acid compositions of the study supplements. The study supplements were identical in appearance and could not be identified on the basis of scent or taste. All study participants were instructed to consume two capsules a day. In addition to consumption of the capsules, participants were advised to follow their normal diet during pregnancy.

\section{Fatty acid composition}

Total fatty acids in plasma, RBCs, and breast milk were extracted by a modified Folch extraction and derivatised with methanolic hydrochloric acid. Fatty acid methyl esters were analysed by gas chromatography/mass spectrometry. The results were expressed in both relative (percentage of total fatty acids, \% TFA) and absolute (concentrations, nmol/ml) terms.

\section{Infant anthropometry}

Infant anthropometry, including weight, length, and occipital frontal head circumference, was recorded within the first 24 
Table 2 Fatty acid composition of the fish oil (Marinol D40) and the high oleic acid sunflower oil placebo study supplements

\begin{tabular}{lll}
\hline Fatty acid & $\begin{array}{l}\text { Fish oil } \\
\text { (Marinol D40) }\end{array}$ & $\begin{array}{l}\text { Sunflower oil } \\
\text { placebo }\end{array}$ \\
\hline C1 14:0 & 3.7 & \\
C16:0 & 6.7 & 4.0 \\
C16:1n-7 & 4.3 & \\
C18:0 & 2.4 & 5.0 \\
C18:1n-9 & 15.6 & 81 \\
C18:2n-6 & 1.2 & 7.2 \\
C18:3n-3 & 0.8 & \\
C18:4 & 1.4 & \\
C20:0 & 0.1 & \\
C20:1 & 2.0 & \\
C20:5n-3 & 7.2 & \\
C22:1 & 2.7 & \\
C22:5n-6 & 4.1 & \\
C22:6n-3 & 40.4 & \\
Sum of above & 92.6 & 97.2 \\
Others & 9.4 & 3.0 \\
\hline
\end{tabular}

Values are $\%$ total fatty acids.

hours after delivery and at both follow up vision tests. Weight was measured using a calibrated Seca Baby Balance (Seca, model 724, Hamburg, Germany) and recorded to the nearest $g$. Length was measured in the supine position using an infant measuring mat and recorded to the nearest $0.5 \mathrm{~cm}$. The largest occipital frontal head circumference was recorded to the nearest $0.1 \mathrm{~cm}$ using a paper measuring tape. Standard deviation scores and centiles were determined using the British 1990 Growth Reference Program (Child Growth Foundation, 1996, London, UK). This allowed anthropometric measurements to be standardised for sex differences.

\section{Visual assessments}

Both flash and pattern-reversal VEPs were recorded under transient conditions using a PC based stimulus and recording system designed by the Department of Clinical Physics and Bio-Engineering, West of Scotland Health Boards. ${ }^{32}$ VEPs were recorded from a midline occipital electrode. A ground electrode was placed on the mastoid, and a midline frontal electrode was used as a reference. Scalp-electrode impedance was measured before each test using an impedance meter. Impedances were normally below $5 \mathrm{kOhms}$ but always below $10 \mathrm{kOhms}$ and approximately equal across electrodes. Electrode leads were positioned close together and secured away from other electrical equipment to minimise electrical interference. Signals were amplified and filtered by a Biodata PA400 amplifier (Biodata, Manchester, UK) with a bandwidth of $0.5-100 \mathrm{~Hz}$ and a gain of 5000. The signal was then passed to the PC system. Automatic artefact rejection was used to reject trials with excessive artefact $(> \pm 100 \mu \mathrm{V})$ that may be due to background electroencephalographic activity, muscle artefact, or environmental noise.

A Grass PS33 Plus photostimulator (Astro-Med Inc, West Warwick, Rhode Island, USA) was used to deliver the flash stimuli. This photostimulator was calibrated with an integrating photometer (International Light IL1700, Newburyport, Massachusetts, USA), and the flash intensity was $1376 \mathrm{~cd} . \mathrm{s} / \mathrm{m}^{2}$ (Grass intensity 16). The infants were either held by the parent or researcher or placed supine in a cot, and the hand held strobe light was then presented $20 \mathrm{~cm}$ from the infants' eyes and stimulated at a rate of 1.1 flashes per second. Between 30 and 60 presentations per trial were averaged over a 500 millisecond epoch. Two or more trials at each stimulus were recorded to ensure reliability and reproducibility of the waveform. The main characteristics of the flash VEP waveform investigated were the peak latencies and the number of com- ponents present. Amplitudes are not reported as they have larger intersubject variability compared with peak latencies. ${ }^{33}$ At the term age test only, flash VEPs were recorded after an electroretinogram and therefore pupils had been dilated. Also, sleep state was assessed at the term age test as the infants were in a variety of states during testing compared with the follow up tests when they were all awake.

Stimuli for recording pattern-reversal VEPs consisted of high contrast black and white checks generated on a colour IBM monitor. The dimensions of the monitor screen measured $27.5 \mathrm{~cm}$ horizontally by $20 \mathrm{~cm}$ vertically so that visual field subtended to $30 \times 24^{\circ}$ at a viewing distance of $43 \mathrm{~cm}$. The mean luminance of the screen was $60 \mathrm{~cd} / \mathrm{m}^{2}$, and the contrast between the black and white checks was greater than $90 \%$. Before recording, the screen luminance was calibrated using a Minolta luminance meter (LS-100). The checkerboard pattern was reversed at a rate of 2.2 reversals per second $(1.1 \mathrm{~Hz})$.

Pattern-reversal VEPs were only recorded when the infant was alert and attentive. The infant was seated on a parent's lap $43 \mathrm{~cm}$ away from and level with the stimulus monitor. Recording pattern-reversal VEPs required two experienced testers. One tester, located behind the stimulus monitor, attracted the infant's attention towards the screen and monitored fixation by making sounds and jingling keys or small toys. The other tester operated the computer. Recording was stopped when the infant was not fixating on the stimulus so that the computer only acquired data during those periods when the infant was viewing the stimulus.

For a subject-to-screen distance of $43 \mathrm{~cm}$, check sizes from 120 to 6 minutes were available. The first size of stimulus presented to each infant was a 120 minute pattern-reversal checkerboard. If there was a clearly reproducible VEP to the 120 minute checks, smaller checks $(60,30,24,12$, and 6 minutes) were presented in an arbitrary order until the signal was absent or until cooperation was lost. A minimum of 30 and maximum of 50 epochs were averaged for each trial. The main characteristics of the pattern-reversal VEP waveform investigated were the peak latencies and amplitudes of the main components, and threshold check size. Threshold check size was defined as the smallest check size to which a reproducible and/or clearly recognisable pattern-reversal VEP was elicited. ${ }^{34}$ To meet the criteria, subjects either failed to show a reproducible response to smaller checks or achieved a reproducible response to the 6 minute checks.

\section{Sample size and statistical analysis}

A sample size of 21 infants per maternal supplementation group would be necessary to detect a one standard deviation difference in the pattern-reversal VEP P100 peak latency (20 milliseconds), with a significance level of $\mathrm{p}<0.05$ and power of $90 \%$. We estimated a $20 \%$ drop out at each time point, and there were five time points (week 15 of pregnancy, week 28 of pregnancy, term, 50 weeks PCA, 66 weeks PCA). Therefore, to allow for loss to follow up, we aimed to recruit 50 mother-infant pairs per supplementation group.

Group means, for maternal and infant characteristics, were compared using a one way analysis of variance for continuous variables and a $\chi^{2}$ for categorical variables. Comparisons of anthropometric data between supplementation groups were made using a repeated measures analysis of variance, with age at testing and supplementation group as the main factors. Differences in mean VEP peak latencies and/or amplitudes among supplementation groups were determined using repeated measures analysis of variance, with age at testing and supplementation group as the main factors. A $\chi^{2}$ test was used to check for any associations between number of components in the flash VEP waveform and supplementation group. Differences in the pattern-reversal VEP threshold check sizes and supplementation group were tested using Kendall's tau $(\tau)$. Multiple linear regression analysis was used to relate the RBC and plasma DHA composition with VEP peak latencies and/or amplitudes. 
Table 3 Characteristics of infants in the maternal fish oil and placebo supplemented groups

\begin{tabular}{lll}
\hline Characteristic & Fish oil & Placebo \\
\hline Gestational age at birth (days) & $279.7(9.5)$ & $279.6(8.5)$ \\
Male/female ratio at birth & $16 / 15$ & $11 / 18$ \\
Birth weight (g) & $3507.7(500.8)$ & $3645.1(495)$ \\
Birth length (cm) & $52.6(2.5)$ & $53.1(2.9)$ \\
Birth head circumference (cm) & $34.9(1.5)$ & $34.9(1.5)$ \\
Male/female ratio at 50 weeks PCA & $15 / 12$ & $11 / 17$ \\
PCA at 50 weeks PCA vision test (days) & $349.7(6.5)$ & $348.3(4.7)$ \\
Weight at 50 weeks PCA vision test (kg) & $5894.4(662.3)$ & $5995.7(827.9)$ \\
Length at 50 weeks PCA vision test (cm) & $60.0(2.6)$ & $60.5(2.9)$ \\
Head circumference at 50 weeks PCA vision test (cm) & $39.9(1.5)$ & $40.1(2.3)$ \\
Male/female ratio at 66 weeks PCA & $16 / 12$ & $11 / 16$ \\
PCA at 66 weeks PCA vision test (days) & $465.4(11.9)$ & $466.7(10.5)$ \\
Weight at 66 weeks PCA vision test (kg) & $8263.7(999.4)$ & $8626.7(208.8)$ \\
Length at 66 weeks PCA vision test (cm) & $68.5(2.6)$ & $69.1(3.2)$ \\
Head circumference at 66 weeks PCA vision test (cm) & $43.8(2.4)$ & $44.1(1.7)$ \\
Mode of feeding at birth (breast/formula) & $20 / 11$ & $21 / 8$ \\
Mode of feeding at 50 weeks PCA (breast/formula/combined) & $9 / 15 / 4$ & $6 / 13 / 8$ \\
Mode of feeding at 66 weeks PCA (breast/formula/combined) & $3 / 19 / 6$ & $2 / 20 / 5$ \\
\hline Results are expressed as mean (SD). & & \\
PCA, Postconceptional age. & &
\end{tabular}

Normally distributed data were expressed as means (SD), and data that were not normally distributed were expressed as medians and ranges. Results were considered significant if $\mathrm{p}<0.05$. All statistical analyses were performed using SPSS for Windows (version 9.0; SPSS Inc, Chicago, Illinois, USA).

\section{RESULTS}

\section{Subjects}

Of the 100 women recruited to the study at 15 weeks of pregnancy (mean 15.4 weeks/109 days; range 14.6-17 weeks/104119 days), 29 withdrew from the study before their routine antenatal clinic appointment at 28 weeks of pregnancy and a further seven withdrew between week 28 and delivery. The most common reasons for withdrawing were poor compliance in capsule consumption $(\mathrm{n}=16)$ and frequent nausea/ vomiting episodes $(n=13)$. Other reasons for withdrawal included loss of contact $(n=3)$, anxiousness $(n=2)$, and unspecified $(\mathrm{n}=3)$. Sixty three women remained in the study at delivery.

Participants in the supplemented and placebo groups did not differ significantly in terms of maternal age, previous obstetric history, socioeconomic status, smoking habits and alcohol intake, exercise patterns, and dietary intake of fish at admission. Furthermore, there were no significant differences in dietary intake of fish or consumption of dietary supplements containing DHA between supplementation groups at any point during the study period.

Of the 63 women remaining in the study at delivery, only 60 of their infants were eligible to continue in the postnatal portion of the study. Infants born before 36 weeks gestation $(\mathrm{n}=1)$ and those with an Apgar score of less than 7 at five minutes $(n=2)$ were excluded. Birth vision tests were performed on 59 of the 60 infants, as one infant, in the fish oil group, was discharged from hospital before the testing session could be arranged. Follow up vision testing was attended by 57 of the 60 infants remaining in the study at birth. One infant, in the fish oil group, was unable to attend the 50 weeks PCA vision test but returned for the 66 weeks PCA test. One infant, in the placebo group, attended the 50 weeks PCA vision test but was unable to attend the 66 weeks PCA test. Therefore 55 mothers and infants, 28 in the fish oil and 27 in the placebo group, attended all three testing sessions and thus completed the entire study.

There were no significant differences between infants in the fish oil and placebo maternal supplementation groups in terms of gestational age at birth, male/female ratio, PCA at the time of vision testing, or mode of feeding (table 3 ).

\section{Fatty acid composition}

The fish oil and placebo groups did not differ in maternal RBC and plasma fatty acid composition at enrollment. Both groups exhibited an increase in maternal RBC and plasma DHA status (\% TFA and concentration) between baseline and week 28 of pregnancy. By 28 weeks, DHA accounted for a significantly higher \% TFA in RBC $(p<0.05)$ and a higher concentration in both RBC and plasma $(\mathrm{p}<0.05)$ in the fish oil compared with placebo group. At term delivery, RBC and plasma DHA had declined relative to 28 week DHA status in all mothers. The \% TFA and concentration $(\mathrm{p}<0.05)$ in maternal RBCs remained higher in the fish oil than placebo group (table 4). There were no significant differences between infants in the fish oil and placebo maternal supplementation groups in DHA as a concentration or \% TFA measured from cord blood (table 5). Finally, there were no significant differences in DHA as a concentration or \% TFA measured in breast milk at $2-5$ postnatal days between the groups. The median concentration of DHA in the breast milk samples of the fish oil and placebo groups were $0.2 \mathrm{nmol} / \mathrm{ml}$ (range $0.1-1.1$ ) and $0.3 \mathrm{nmol} / \mathrm{ml}(0.1-0.5$ ) respectively. The relative levels of DHA in the breast milk samples of the fish oil and placebo groups were $2561 \%$ TFA (range 669-16 466) and 1614\% TFA (260-6000) respectively.

\section{Infant anthropometry}

There were no significant differences in mean weight, length, or head circumference between infants in the fish oil supplemented and control group at any of the assessment times. Moreover, there were no significant differences in standard deviation scores or centiles between supplementation groups. Infants in the lowest quartile for cord blood DHA (concentration and \% TFA) did not differ significantly from those in the highest quartile for weight, length, or head circumference at any of the assessment times.

\section{Visual assessments}

The mean peak latency of the major components of the flash VEP waveform (N1, P1, N2, P2, N3) did not differ significantly between infants in the fish oil and placebo supplemented group (table 6). As expected, there was a significant shortening of the mean peak latency of the P1 $(p<0.0001), N 2$ $(p<0.0001)$, P2 $(p<0.0001)$, and N3 (p<0.005) components with age, regardless of supplementation group. The 
Table 4 Absolute $(\mathrm{nmol} / \mathrm{ml})$ and relative (\% TFA) measures of fatty acids in maternal red blood cells (RBCs) and plasma at birth

\begin{tabular}{|c|c|c|}
\hline Fatty acids & Fish oil $(n=29)^{*}$ & Placebo $(n=25)^{*}$ \\
\hline \multicolumn{3}{|c|}{ RBCs (nmol/ml) } \\
\hline Total n-6 & 639 (131-1065) & $623(151-1040)$ \\
\hline $\mathrm{AA}$ & 225 (19-393) & 243 (49-372) \\
\hline Total n-3 & $207(9-393)$ ** & 151 (24-298) \\
\hline DHA & $3(0.3-5)^{* *}$ & $2(0.5-4)$ \\
\hline EPA & $0(0-19)$ & $0(0-13)$ \\
\hline \multicolumn{3}{|l|}{ RBCs (\% TFA) } \\
\hline Total n-6 & 19.8 (9.7-22.3) & $19.6(13.0-23.1)$ \\
\hline $\mathrm{AA}$ & $8.5(1.8-10.5)$ & $9.2(3.9-11.5)$ \\
\hline Total $n-3$ & $3.9(0.5-6.3)^{* *}$ & $3.3(0.7-5.3)$ \\
\hline $\mathrm{DHA}$ & $168.2(6.2-305.3)^{* *}$ & $118.9(17.3-225.8)$ \\
\hline EPA & $0.0(0.0-0.3)$ & $0.0(0.0-0.2)$ \\
\hline \multicolumn{3}{|c|}{ Plasma (nmol/ml) } \\
\hline Total $n-6$ & 2567 (1025-4107) & 2384 (1597-4817) \\
\hline $\mathrm{AA}$ & $245(107-455)$ & $266(163-531)$ \\
\hline Total $n-3$ & $282(29-2184)$ & $243(82-510)$ \\
\hline $\mathrm{DHA}$ & $172(29-1954)$ & $139(61-346)$ \\
\hline EPA & $24(0-91)$ & $19(0-61)$ \\
\hline \multicolumn{3}{|c|}{ Plasma (\% TFA) } \\
\hline Total n-6 & 30.8 (24.5-36.9) & $29.6(23.3-34.5)$ \\
\hline $\mathrm{AA}$ & $3.7(1.9-6.3)$ & $4.0(2.9-5.3)$ \\
\hline Total $n-3$ & $2.4(0.2-3.7)$ & $2.0(0.8-3.9)$ \\
\hline $\mathrm{DHA}$ & $1.5(0.2-2.2)$ & $1.3(0.6-2.6)$ \\
\hline EPA & $0.2(0.0-0.6)$ & $0.2(0.0-0.6)$ \\
\hline
\end{tabular}

Values are median (range).

*Blood samples were not obtained from four subjects in the fish oil group and two subjects in the placebo group at birth. * *Significantly higher than placebo group.

AA, Arachidonic acid; DHA, docosahexaenoic acid; EPA eicospentaenoic acid; \% TFA, percentage of total fatty acids.

Table 5 Absolute $(\mathrm{nmol} / \mathrm{ml})$ and relative (\% TFA) measures of fatty acids in umbilical cord red blood cells (RBCs) and plasma at birth

\begin{tabular}{lll}
\hline Fatty acids & Fish oil $(n=27)^{*}$ & Placebo $(n=26)^{*}$ \\
\hline RBCs $(n m o l / m l)$ & & \\
Total $n-6$ & $1150(613-2121)$ & $1397(480-3086)$ \\
AA & $349(239-734)$ & $422(154-690)$ \\
Total $n-3$ & $255(70-481)$ & $301(104-542)$ \\
DHA & $221(70-465)$ & $273(98-478)$ \\
EPA & $2(0-13)$ & $2(0-41)$ \\
RBCs $(\%$ TFA) & & \\
Total $n-6$ & $20.8(16.7-23.1)$ & $21.5(14.0-23.8)$ \\
AA & $12.4(9.2-14.5)$ & $12.5(8.2-14.3)$ \\
Total $n-3$ & $4.6(1.7-6.2)$ & $4.8(1.8-7.1)$ \\
DHA & $4.4(1.7-5.8)$ & $4.2(1.8-6.2)$ \\
EPA & $0.0(0.0-0.1)$ & $0.0(0.0-0.3)$ \\
Plasma $(n m o l / m l)$ & & \\
Total $n-6$ & $519(151-1654)$ & $581(61-2601)$ \\
AA & $147(11-204)$ & $166(18-260)$ \\
Total $n-3$ & $467(40-1384)$ & $431(5-1346)$ \\
DHA & $460(40-1335)$ & $413(5-1299)$ \\
EPA & $8(0-15)$ & $5(0-15)$ \\
Plasma $(\%$ TFA) & & \\
Total $n-6$ & $25.8(17.8-31.6)$ & $27.0(20.0-31.9)$ \\
AA & $10.6(1.9-14.3)$ & $11.2(4.1-14.8)$ \\
Total $n-3$ & $3.3(0.4-6.8)$ & $3.4(1.1-6.2)$ \\
DHA & $3.0(0.4-6.0)$ & $3.0(1.0-5.1)$ \\
EPA & $0.3(0.0-1.1)$ & $0.2(0.0-0.8)$ \\
\hline
\end{tabular}

Values are median (range)

* Blood samples were not obtained from four infants in the fish oil group and three infants in the placebo group at birth.

AA, Arachidonic acid; DHA, docosahexaenoic acid; EPA eicospentaenoic acid; \% TFA, percentage of total fatty acids.

number of components in the flash VEP waveform did not differ between supplementation groups at birth or 50 or 66 weeks PCA. No significant correlations were observed between flash VEP peak latencies and RBC or plasma DHA levels in
Table 6 Mean peak latencies of the major components of the transient flash visual evoked potential waveform for infants in the maternal fish oil and placebo supplemented groups at each of the three assessment ages

\begin{tabular}{|c|c|c|c|}
\hline Test age & Component & Fish oil group & Placebo group \\
\hline Birth & $\begin{array}{l}\text { N1 } \\
\text { P1 } \\
\text { N2 } \\
\text { P2 } \\
\text { N3 }\end{array}$ & $\begin{array}{l}n=5 \\
62.2(3.8) \\
n=9 \\
101(13.6) \\
n=27 \\
153.5(28.9) \\
n=28 \\
201.9(28.4) \\
n=26 \\
292.2(58.2)\end{array}$ & $\begin{array}{l}n=4 \\
74.8(16.8) \\
n=5 \\
107.8(11.8) \\
n=22 \\
149.9(28.0) \\
n=27 \\
201.8(33.3) \\
n=27 \\
298.4(52.8)\end{array}$ \\
\hline 50 weeks PCA & $\begin{array}{l}\text { N1 } \\
\text { P1 } \\
\text { N2 } \\
\text { P2 } \\
\text { N3 }\end{array}$ & $\begin{array}{l}n=19 \\
54.7(16.2) \\
n=23 \\
80.3(21.1) \\
n=24 \\
128.9(47.9) \\
n=21 \\
164.2(29.9) \\
n=14 \\
241.8(49.8)\end{array}$ & $\begin{array}{l}n=18 \\
58.1(21.4) \\
n=22 \\
84.2(22.5) \\
n=28 \\
112.8(46.5) \\
n=26 \\
162.5(26.5) \\
n=20 \\
277.3(49.4)\end{array}$ \\
\hline 66 weeks PCA & $\begin{array}{l}\text { N1 } \\
\text { P1 } \\
\text { N2 } \\
\text { P2 } \\
\text { N3 }\end{array}$ & $\begin{array}{l}n=23 \\
61.5(5.4) \\
n=25 \\
80.1(15.8) \\
n=25 \\
128.5(30.3) \\
n=12 \\
150.6(33.0) \\
n=11 \\
228.9(55.9)\end{array}$ & $\begin{array}{l}n=24 \\
57.3(10.7) \\
n=26 \\
76.5(19.5) \\
n=26 \\
122.1 \text { (33.7) } \\
n=19 \\
152.5(43.6) \\
n=15 \\
209.2(38.2)\end{array}$ \\
\hline
\end{tabular}

infant cord blood at any time point. Moreover, maternal breast milk DHA levels measured shortly after birth did not correlate with flash VEP peak latencies.

Pattern-reversal VEPs showed the expected early rapid maturation, with shortening of the peak latencies, increasing amplitude, and a gradual sharpening of the waveform. The peak latency of the P100 component of the pattern-reversal VEP did not differ significantly between infants in the fish oil supplemented and placebo group for any of the check sizes at either 50 or 66 weeks PCA (fig 1). Moreover, there were no significant differences between the fish oil and placebo group in the pattern-reversal VEP threshold check size reached at either 50 or 66 weeks PCA. Interestingly, despite a lack of difference in P100 peak latencies between maternal supplementation groups, there was a significant correlation between infant cord RBC DHA expressed in both absolute $(\mathrm{nmol} / \mathrm{ml})$ and relative measures (\% TFA) and pattern-reversal VEP peak latencies. Multiple regression analyses revealed that the independent variables predicting P100 peak latencies included PCA at the time of assessment, sex, check size, and umbilical cord RBC DHA status. PCA predicted P100 peak latencies, with older PCA at the time of assessment resulting in shorter peak latencies. Sex was significantly associated with the P100 component, with girls showing shorter peak latencies than boys. ${ }^{35}$ As expected, check size predicted P100 peak latencies, with longer latencies observed for smaller check sizes. In addition, there was a significant negative correlation between umbilical cord RBC DHA (nmol/ml and \% TFA) and pattern-reversal VEP peak latencies (tables 7 and 8). In other words, a higher RBC DHA status at birth is associated with earlier VEP development, as evidenced by shorter peak latencies. Measures of 


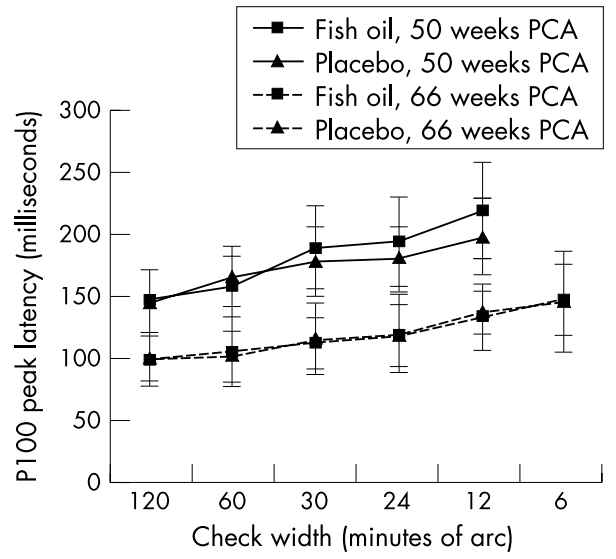

Figure 1 Peak latency of the pattern-reversal visual evoked potential recorded at 50 and 66 weeks post-conceptional age (PCA) in infants born to mothers supplemented with or without fish oil during pregnancy. The main effects of age group and check size are evident ( $p<0.001)$, but significant differences based on maternal supplementation group (fish oil, placebo) by repeated measures analysis of variance were not observed. Values are means and bars represent SD.

Table 7 Multiple regression results for the independent variables predicting the peak latency of the P100 component

\begin{tabular}{llll}
\hline Variable & $B$ & $\beta$ & $\mathrm{p}$ Value \\
\hline RBC DHA (nmol/ml) & $-3.35 \mathrm{E}-02$ & -0.07 & 0.03 \\
Check size & 18.87 & 0.38 & 0.0001 \\
PCA (days) & -0.50 & -0.73 & 0.0001 \\
Sex & -14.36 & -0.18 & 0.0001 \\
\hline
\end{tabular}

An $R$ of 0.808 was obtained $\left(F_{(4,323)}=151.91, p<0.0001\right)$. Infant umbilical cord DHA status is expressed as $R B C$ concentration $(\mathrm{nmol} / \mathrm{ml})$.

$B$, Unstandardised regression coefficient (slope); $\beta$, standardised coefficient; $p$, significance level; RBC, red blood cell; DHA docosohexaenoic acid; PCA, postconceptional age.

Table 8 Multiple regression results for the independent variables predicting the peak latency of the P100

\begin{tabular}{llll}
\hline Variable & $B$ & $\beta$ & $p$ Value \\
\hline RBC DHA (\% TFA) & -4.61 & -0.09 & 0.004 \\
Check size & 10.89 & 0.38 & 0.0001 \\
PCA (days) & -0.50 & -0.74 & 0.0001 \\
Sex & -14.96 & -0.18 & 0.0001 \\
\hline
\end{tabular}

An $R$ of 0.810 was obtained $\left(F_{(4,323)}=154.52, p<0.0001\right)$. Infant umbilical cord DHA status is expressed as $R B C$ concentration $(\mathrm{nmol} / \mathrm{ml})$.

$B$, Unstandardised regression coefficient (slope); $\beta$, standardised coefficient; $p$, significance level; RBC, red blood cell; DHA, docosohexaenoic acid; PCA, postconceptional age.

cord blood plasma were not significant predictors of P100 peak latencies. However, there was a trend towards shorter P100 peak latencies with higher plasma DHA concentrations $(p=0.12)$. Finally, maternal breast milk DHA levels measured shortly after birth did not correlate with pattern-reversal VEP peak latencies.

The median value for RBC DHA status (\% TFA) of infants in the top quartile were on average $2 \%$ higher than the median value for infants in the lowest quartile (table 9). Multiple regression analyses revealed that the P100 peak latency shortens by approximately 0.5 milliseconds a day between 50 and 66 weeks PCA. Thus maturation of the P100 peak latency for
Table 9 Quartiles for DHA in umbilical cord RBC $1 \%$ TFA and $\mathrm{nmol} / \mathrm{ml}$ )

\begin{tabular}{lll}
\hline $\begin{array}{l}\text { Umbilical cord blood } \\
\text { quartiles }\end{array}$ & $\begin{array}{l}\mathrm{RBC} \text { concentration } \\
\text { (nmol/ml) }\end{array}$ & $\mathrm{RBC}(\% \mathrm{TFA})$ \\
\hline Quartile 1 - Low & $175.7(97.6-187.2)$ & $3.45(1.77-3.81)$ \\
Quartile 2 - Medium 1 & $212.9(188.8-240.4)$ & $4.08(3.84-4.27)$ \\
Quartile 3 - Medium 2 & $281.7(254.0-303.2)$ & $4.54(4.3-4.8)$ \\
Quartile 4 - High & $353.2(321.2-477.9)$ & $5.46(5.01-6.16)$ \\
\hline
\end{tabular}

Values are median (range)

DHA, Docosahexaenoic acid; RBC, red blood cell; \% TFA, percentage of total fatty acids.

infants in the highest quartile for RBC DHA status (\% TFA) at birth was advanced by an average of 20 days compared with infants in the lowest quartile.

\section{DISCUSSION}

The n-3 LCPUFA, DHA, is present in large proportions in the grey matter of the cerebral cortex of the brain where it is believed to play an important role in regulating the fluidity of cell membranes and thus the efficiency of neuroelectrical signal transmission. ${ }^{36}$ The presence of DHA is particularly important during early development of the central nervous system. The rapid accumulation of DHA in the central nervous system during the final trimester of pregnancy and the initial months of life occurs in harmony with the process of synaptogenesis, the formation of vast numbers of synaptic connections between nerve cells. ${ }^{47}$ The flash and pattern-reversal VEPs are electrophysiological tests that measure the electrical field generated after transmission by neurons along the primary visual pathway initiated by changing visual stimuli; they provide a reliable assessment of the integrity of the central visual pathways. It is hypothesised that a deficiency in DHA during development may be evidenced in delayed maturation of the VEP. Recent clinical trials have used the VEP to investigate whether a postnatal dietary supply of DHA is important for optimal visual development in both preterm ${ }^{18} 2038$ and term infants. ${ }^{21-23}$

We were unable to identify any advantageous effects of maternal dietary fish oil supplementation during pregnancy on maturation of the VEP. Flash and patternreversal VEP development during the first 6 months of life was comparable between infants born of mothers supplemented with a source of DHA and infants born of mothers who were not supplemented. The lack of a significant difference in VEP maturation between infants in the maternal fish oil supplemented and placebo group may be explained in part by the natural physiological process whereby the fetus obtains the essential nutrients from its mother during gestation depleting her stores. Infants in both groups had sufficient amounts of DHA to support normal VEP development, and no gross differences were observed between groups.

There are few published studies with which to compare our findings, as most investigations into LCPUFAs and visual development have supplemented infant diets or compared breast fed infants with those who were formula fed. A recent similar randomised controlled trial, supplementing women with cod liver oil during pregnancy and for the first three months post partum, reported no beneficial effect on infant cognitive development as measured by the novelty preference test (Fagan test) at 6 and 9 months of age. ${ }^{39}$ In contrast with our findings, despite no obvious functional benefit to the infants, an increase in infant umbilical plasma DHA in the maternal cod liver oil supplemented group relative to the placebo group was reported. This increase in infant DHA status may have been the result of a seven times higher content of DHA in the cod liver oil supplement compared with our 
blended fish oil supplement. It was somewhat surprising, however, that we did not observe an increased DHA status in infants at birth, as previous studies have reported elevated DHA levels in the umbilical plasma and/or RBCs of term infants in relation to DHA supplemented maternal diets. ${ }^{11} 4041$ However, although maternal diet is a contributing factor, it may not be the only determinant of neonatal fatty acid status, as genetic or other factors may ensure that the maternal to fetal supply is adequate. ${ }^{42}$

Although maternal fish oil supplementation did not elevate DHA status of the infants at birth, the actual DHA status in umbilical cord RBCs, irrespective of maternal supplementation group, was associated with maturation of the P100 component of the pattern-reversal VEP. Across the full data set, after correcting for sex, age, and check size, peak latency of the P100 component was 4.6 milliseconds shorter for infants with a $1 \%$ higher DHA status (\% TFA) in umbilical cord blood at birth. Moreover, infants in the upper quartile for RBC DHA (\% TFA) status showed pattern-reversal VEPs that were more mature than infants in the lowest quartile. Precisely, P100 peak latencies of the pattern-reversal VEP were advanced by nearly three weeks in infants in the upper quartile for RBC DHA (\% TFA).

No association between DHA status and maturation of the flash VEP was detected in this study. In comparison with the pattern-reversal VEP, the flash VEP has a greater degree of variability and a wider range of normal values, and therefore does not provide as sensitive a measure of visual pathway function..$^{43}$ Taylor et al suggest that the flash VEP may not be sufficiently sensitive for detection of the subtle effects of DHA on maturation of the visual pathways.

Our study supports the essentiality of the LCPUFA, DHA, during fetal development. Our results suggest that term infants accrue sufficient amounts of DHA in spite of and/or at the expense of their mothers, while in utero, to meet the developing needs of the neural and visual systems, so that no direct benefit of maternal supplementation was observed. However, our results did show a relation between infant RBC DHA status at birth and maturation of the pattern-reversal VEP at 50 and 66 weeks PCA, suggesting that infant DHA status itself may influence visual pathway maturation. Although all of the infants in this study had pattern-reversal peak latencies within normal ranges, those with higher concentrations and relative amounts of DHA in umbilical cord RBCs at birth showed more mature VEPs at 50 and 66 weeks PCA. This finding suggests that higher DHA status in RBCs at birth may indicate better availability of DHA for incorporation within synaptic membranes. This may contribute to more efficient transmission of neuroelectrical signals and result in shorter peak latencies, an indicator of more mature VEPs. Further research is required to determine whether the differences in maturation of the pattern-reversal VEP detected at 50 and 66 weeks PCA persist or contribute to any long term advantage. In addition, the physiological processes determining how some infants acquire higher proportions of $n-3$ LCPUFAs in cord blood at birth than others remain unknown. The results of this study suggest that there are factors in addition to increased maternal dietary intake during pregnancy that contribute to neonatal DHA status.

We gratefully acknowledge the financial support of the Chief Scientist's Office, Scottish Office Health Department (Grant K/MRS/ 50/C2730). We thank R P Scherer Limited for their donation of the both the fish oil and placebo capsules. We are grateful to Ms Ruth Hamilton and Dr Helen Mactier for their assistance with the VEP recordings and analysis. Most importantly, we would like to thank all the mothers and infants who participated in this study.

\section{Authors' affiliations}

C A Malcolm, D L McCulloch, Department of Vision Sciences, Glasgow Caledonian University, Glasgow, Scotland, UK

C Montgomery, L T Weaver, Department of Child Health, University of Glasgow, Royal Hospital for Sick Children, Glasgow, Scotland, UK A Shepherd, Department of Nursing and Midwifery, University of Stirling, Stirling, Scotland, UK

\section{REFERENCES}

1 Innis SM. Essential fatty acids in growth and development. Prog Lipid Res 1991;30:39-103.

2 Svennerholm J. Distribution and fatty acid composition of phosphoglycerides in normal human brain. J Lipid Res 1968;9:570-9.

3 Anderson RE, Maude MB, Zimmerman W. Lipids of ocular tissues. X. Lipid composition of subcellular fractions of bovine retina. Vision Res 1975; 15:1087-90

4 Clandinin MT, Chappell JE, Leong S, et al. Intrauterine fatty acid accretion rates in human brain: implications for fatty acid requirements Early Hum Dev 1980;4:121-9.

5 Farquharson J, Jamieson EC, Logan RW, et al. Docosahexaenoic and nervonic acids in term and preterm infant cerebral white matter. Prenat Neonatal Med 1996;1:234-40.

6 Carnielli VP, Wattimena DJ, Luijendijk IH, et al. The very low birth weight premature infant is capable of synthesizing arachidonic and docosahexaenoic acids from linoleic and linolenic acids. Pediatric Res 1996:40: 169-74

7 Szitanyi P, Koletzko B, Mydlilova A, et al. Metabolism of ${ }^{13} \mathrm{C}$-labeled linoleic acid in newborn infants during the first week of life. Pediatric Res 1999;45:669-73

8 Larque E, Demmelmair H, Koletzko B. Perinatal supply and metabolism of long-chain polyunsaturated fatty acids: importance for the early development of the nervous system. Ann NY Acad Sci 2002;967:299-310.

9 Al MDM, van Houwelingen AC, Kester ADM, et al. Maternal essential fatty acid patterns during normal pregnancy with their relationship to the neonatal essential fatty acid status. Br J Nutr 1995;74:55-68.

10 Sattar N, Gaw A, Stephens IFD, et al. Pregnancy associated changes in plasma and erythrocyte membrane essential fatty acids [abstract]. Proceedings of the 2nd International Society for the Study of Fatty Acids and Lipids (ISSFAL) 7-10 June 1995, Washington, p 54.

11 van Houwelingen AC, Sorenson JDJ, Hornstra G, et al. Essential fatty acids in neonates after fish oil supplementation during late pregnancy. $B$ J Nutr 1995;74:723-31.

12 Connor WE, Lowensohn R, Hatcher L. Increased docosahexaenoic acid levels in human newborn infants by the administration of sardines and fish oil during pregnancy. Lipids 1996;31:S183-7.

13 Uauy RD, Birch DG, Birch EE, et al. Effect of dietary omega-3 fatty acids on retinal function of very-low-birth-weight neonates. Pediatric Res 1990;28:485-92.

14 Birch DG, Birch EE, Hoffman DR, et al. Retinal development in very-low-birth-weight infants fed diets differing in omega-3 fatty acids. Invest Ophthalmol Vis Sci 1992:33:2365-76.

15 Carlson SE, Werkman SH, Rhodes PG, et al. Visual-acuity development in healthy preterm infants: effect of marine-oil supplementation. Am J Clin Nutr 1993;58:35-42

16 Carlson SE, Werkman SH. A randomized trial of visual attention of preterm infants fed docosahexaenoic acid until two months. Lipids 1996:31:85-90.

17 Carlson SE, Werkman SH, Tolley EA. Effect of long-chain n-3 fatty acid supplementation on visual acuity and growth of preterm infants with and without bronchopulmonary dysplasia. Am J Clin Nutr 1996;63:687-97.

18 Faldella G, Govoni M, Alessandroni R, et al. Visual evoked potentials and dietary long chain polyunsaturated fatty acids in preterm infants. Arch Dis Child Fetal Neonatal Ed 1996;75:F108-12.

19 Werkman SH, Carslon SE. A randomized trial of visual attention of preterm infants fed docosahexaenoic acid until nine months. Lipids 1996:31:91-7.

20 O'Connor DL, Hall R, Adamkins D, et al. Growth and development in preterm infants fed long-chain polyunsaturated fatty acids: a prospective, randomised controlled trial. Pediatrics 2001;108:359-71.

21 Makrides M, Neumann M, Simmer K, et al. Are long-chain polyunsaturated fatty acids essential nutrients in infancy? Lance 1995;345: 1463-8.

22 Carlson SE, Ford AJ, Werkman SH, et al. Visual acuity and fatty acid status of term infants fed human milk and formulas with and without docosahexaenoate and arachidonate from egg yolk lecithin. Pediatr Res 1996;39:882-7.

23 Birch EE, Hoffman DR, Uauy R, et al. Visual acuity and the essentiality of docosahexaenoic acid and arachidonic acid in the diet of term infants. Pediatr Res 1998; 44:201-8.

24 Auestad N, Montalto MB, Hall RT, et al. Visual acuity, erythrocyte fatty acid composition, and growth in term infants fed formulas with long chain polyunsaturated fatty acids for one year. Pediatr Res 1997;41:1-10.

25 Jorgensen $\mathbf{M H}$, Holmer $\mathrm{G}$, Lund $\mathrm{P}$, et al. Effect of formula supplemented with docosahexaenoic acid and gamma-linolenic acid on fatty acid status and visual acuity in term infants. J Pediatr 1998;26:412-21.

26 Makrides M, Neumann MA, Simmer K, et al. A critical appraisal of the role of dietary long-chain polyunsaturated fatty acids on neural indices of term infants: a randomized, controlled trial. Pediatrics 2000;105:32-8. 
27 Auestad N, Halter R, Hall RT, et al. Growth and development in term infants fed long-chain polyunsaturated fatty acids: a double-masked, randomised, parallel, prospective, multivariate study. Pediatrics $2001 ; 108: 372-81$

28 Berry C, Montgomery C, Sattar N, et al. Fatty acid status of women of reproductive age. Eur J Clin Nutr 2001;55:518-24.

29 Carstairs V, Morris R. Deprivation with health in Scotland. Health Bull 1990:48:162-75.

30 Cleland LG, James M, Neumann MA, et al. Linoleate inhibits EPA incorporation from dietary fish oil supplements in human subjects. Am J Clin Nutr 1992:55:395-9.

31 Makrides M, Neumann MA, Gibson RA. Effect of maternal docosahexaenoic acid (DHA) supplementation on breast milk composition. Eur J Clin Nutr 1996;50:352-7.

32 Bradnam MS, Evans AL, Montgomery DMI, et al. A persona computer-based visual evoked potential stimulus and recording system. Doc Ophthalmol 1994;86:81-93.

33 Harden A. Maturation of the visual evoked potentials. In: Chiarenza GA, Papakostopoulos D, eds. Clinical application of cerebral evoked potentials in medicine. Amsterdam: Excerpta Medica, 1982:41-59.

34 McCulloch DL, Skarf B. Development of the human visual system: monocular and binocular pattern VEP latency. Invest Ophthalmol Vis Sci 1991;32:2372-81.
35 Malcolm CA, McCulloch DL, Shepherd AJ. Pattern-reversal visual evoked potentials in infants: gender differences during early visual maturation. Dev Med Child Neurol 2002;44:345-51.

36 Kurlak LO, Stephenson TJ. Plausible explanations for effects of long chain polyunsaturated fatty acids (LCPUFA) on neonates. Arch Dis Child Fetal Neonatal Ed 1999;80:F148-54

37 Martinez $\mathbf{M}$. Tissue levels of polyunsaturated fatty acids during early human development. J Pediatr 1992;120:S129-38.

38 Birch EE, Birch DG, Hoffman DR, et al. Dietary essential fatty acid supply and visual acuity development. Invest Ophthalmol Vis Sci 1992;33:3242-53.

39 Helland IB, Saugstad OD, Smith L, et al. Similar effects on infants of $n-3$ and $n-6$ fatty acids supplementation to pregnant and lactating women. Pediatrics 2001;108:1-10.

40 Connor WE, Lowensohn R, Hatcher L. Increased docosahexaenoic acid levels in human newborn infants by administration of sardines and fish oil during pregnancy. Lipids 1996:31(suppl):S183-7.

41 Matorras R, Perteagudo L, Sanjurjo P, et al. Intake of long chain w3 polyunsaturated fatty acids during pregnancy and the influence of levels in the mother on newborn levels. Eur J Obstet Gynecol Reprod Biol 1999;83:179-84

42 Hornstra G, Al MDM, Gerrard JM, et al. Essential fatty acid status of neonates born to Invit mothers: comparison with Caucasian neonates and effect of diet. Prostaglandins Leukot Essent Fatty Acids 1992:45: 125-30.

43 Taylor MJ, Saliba E, Laugier J. Use of evoked potentials in preterm neonates. Arch Dis Child Fetal Neonatal Ed 1996;74:F70-6.

This month in the Archives of Disease in Childhood

The following papers appearing in the September 2003 issue of ADC may be of interest to readers of Fetal and Neonatal.

Original article

Performance, treatment pathways, and effects of alternative policy options for screening for developmental dysplasia of the hip in the United Kingdom. C Dezateux, J Brown, R Arthur, J Karnon, A Parnaby

Original article

Efficiency of alternative policy options for screening for developmental dysplasia of the hip in the United Kingdom. J Brown, C Dezateux, J Karnon, A Parnaby, R Arthur

Original article

The economic burden of environmental tobacco smoke in the first year of life. GM Leung, L-M Ho, T-H Lam

Original article

Maternal recall of exclusive breast feeding duration. RM Bland, N C Rollins, G Solarsh, J Van den Broeck, HM Coovadia, for the Child Health Group 